\title{
STRENGTH CHARACTERISTICS OF HDPE CONCRETE
}

\author{
Sudarshan $^{1}$, Manjulavani ${ }^{2}, \mathrm{~V}$ Bhikshma $^{3}$ \\ ${ }^{1}$ ME Student, Lab Asst., Civil Engineering Dept., Osmania University, Hyderabad \\ ${ }^{2}$ Professor of Civil Engineering, JNTU, Hyderabad \\ ${ }^{3}$ Professor of Civil Engineering, Osmania University, Hyderabad
}

\begin{abstract}
In this research work, an attempt is made to investigate high density polyethylene fibre reinforced concrete (HDPE) for M30 grade concrete. In this experimental work the percentage of HDPE considered is 0 to 6\%. A series of trial mixes have been conducted for the investigation to obtain the desired target strength of HDPE concrete with varying percentages. The mechanical properties are evaluated for all percentages. A total of 78 cubes, 39 cylinders and 39 prisms of standard specimens have been considered. The strength property of HDPE concrete is encouraging the results. As the HDPE is non bio degradable waste disposable material is much useful for the concrete works in durability aspects. The flexural capacity of the concrete also very much improved, hence encouraging the research
\end{abstract}

Keywords: HDPE-FRC, NBD, Cement

\section{INTRODUCTION}

\subsection{Objectives}

1. To mix high density polyethylene small pieces in cement concrete.

2. To study the strength properties of the concrete by using HDPE.

3. To improve the strength properties of the concrete by using certain Additives.

\subsection{Composite}

HDPE fibres (high density polyethylene).

Fibre enables to produce a hardened concrete which has:
* Improved surface quality
* Greater impact resistance
* Increased damage resistance

\subsection{Non Bio Degradable Materials}

* Fibre plastics

* Jute plastics

* Textile waste

* Polythene covers

* Plastics covers

* Disposable glass

* Water bottles

* Fiber reinforced plastic sheets

* Cement bags

In present investigation cement bags are used (NBD). CIPET (Central institute of plastics engineers \& technology) certified that the above bags come under HDPE fibers. HDPE fibers:

\subsection{The Properties for HDPE}

Properties: Physical Properties of HDPE fibers CIPET: (Central Institute of Plastic Engineers \& Technology)

\section{TEST RESULTS}

\begin{tabular}{|c|l|c|c|c|}
\hline $\begin{array}{c}\text { Sl. } \\
\text { No. }\end{array}$ & \multicolumn{1}{|c|}{ Test } & Test Method & Result & Unit \\
\hline 1. & Tensile breaking load at yield & IS:9755:1999/IS:1969 & 61.79 & $\mathrm{Kg}$ \\
\hline 2. & Tensile breaking load at break & IS:9755:1999/IS:1969 & 2.78 & $\mathrm{Kg}$ \\
\hline 3. & Tensile elongation break & IS:9755:1999/IS:1969 & 26.91 & $\%$ \\
\hline 4. & Identification & CIPET Method & $\begin{array}{c}\text { High Density } \\
\text { Polyethylene (HDPE) }\end{array}$ & - \\
\hline
\end{tabular}




\subsection{Factors Influencing the Fibre Reinforced}

\section{Concrete}

* Aspect ratio

* Minimum effective length

* Critical length

* Orientation of fibres

* Volume of fibres

* Spacing of fibres

* Size of coarse aggregate

* Workability \& compaction of concrete

\subsection{Applications}

Asbestos: Sheets, Pipes, tiles and corrugated roofing elements, wall linings.

\section{MATERIALS AND PROPERTIES}

\subsection{Constituents \& Characteristics}

\begin{tabular}{|l|c|c|}
\hline \multicolumn{1}{|c|}{ Oxide-Constituent } & \% Content & Percentage \\
\hline Cao (Line) & $60-67$ & 63.9 \\
\hline SiO2 (Silica) & $17-25$ & 22.4 \\
\hline A12 O3 (Alumina) & $3.0-8.0$ & 4.6 \\
\hline Fe2O3 (Iron Oxide) & $0.5-6.0$ & 1.8 \\
\hline MgO (Magnesia) & $0.1-4.0$ & 0.9 \\
\hline Alkalies (K2O,Na2O) & $0.4-1.3$ & 1.98 \\
\hline SO3 (Sulfurtrioxide) & $1.0-3.0$ & \\
\hline
\end{tabular}

\subsection{Physical Properties of O.P.C (53 Grade)-Ultra Tech Brand}

\begin{tabular}{|c|l|c|}
\hline \multicolumn{1}{|c|}{ Sl. No. } & \multicolumn{1}{|c|}{ Properties } & Test Results \\
\hline 1 & Fineness of cement (sieve no.9) & $3.5 \%$ \\
\hline 2 & Specific Gravity & 3.14 \\
\hline 3 & Normal Consistency & $32 \%$ \\
\hline 4 & Initial setting time & 70 minutes \\
\hline 5 & Final setting time $\quad 225$ minutes \\
\hline \multirow{2}{*}{6} & Compressive Strength (3 days) & $33.5 \mathrm{~N} / \mathrm{mm}^{2}$ \\
\cline { 2 - 4 } & Compressive Strength (7 days) & $45.0 \mathrm{~N} / \mathrm{mm}^{2}$ \\
\cline { 2 - 3 } & Compressive Strength (28 days) & $56.8 \mathrm{~N} / \mathrm{mm}^{2}$ \\
\hline
\end{tabular}


3.3 Quantity of Materials required for 1cum of concrete (By volume)

\begin{tabular}{|c|c|c|c|c|}
\hline $\begin{array}{l}\text { Sl. } \\
\text { No. }\end{array}$ & Cement & Fine Aggregate & Coarse Aggregate & Water Content \\
\hline 1. & $400 / 1440$ & $610 / 1710$ & $1141 / 1585$ & $180 / 1000$ \\
\hline 2. & 0.278 & 0.359 & 0.725 & 0.176 \\
\hline 3. & \multicolumn{3}{|r|}{$2 \%$ entrapped air } & 0.02 \\
\hline \multicolumn{4}{|r|}{ Total Volume } & $1.558 \mathrm{~cm}$ \\
\hline
\end{tabular}

Weight of materials required for 1 batch(30kg)Mix

\begin{tabular}{|c|c|c|c|c|}
\hline $\begin{array}{c}\text { Sl. } \\
\text { No. }\end{array}$ & Cement & Fine Aggregate & Coarse Aggregate & Water Content \\
\hline 1. & $30 / 2.87$ & $1.534 \times 10.45$ & $2.87 \times 10.45$ & $0.44 \times 10.45$ \\
\hline 2. & 10.45 & 16.03 & 30.0 & 4.6 \\
\hline
\end{tabular}

\begin{tabular}{|c|c|c|}
\hline $\begin{array}{c}\text { Sl. } \\
\text { No. }\end{array}$ & \% OF HDPE & Wt of HDPE (gm/cc) \\
\hline 1 & 0.5 & 10.6 \\
\hline 2 & 1.0 & 21.2 \\
\hline 3 & 1.5 & 31.8 \\
\hline 4 & 2.0 & 42.4 \\
\hline 5 & 2.5 & 53.00 \\
\hline 6 & 3.0 & 63.6 \\
\hline 7 & 3.5 & 74.2 \\
\hline 8 & 4.0 & 84.8 \\
\hline 9 & 4.5 & 95.4 \\
\hline 10 & 5.0 & 106.0 \\
\hline 11 & 5.5 & 116.6 \\
\hline 12 & 6.0 & 127.2 \\
\hline
\end{tabular}

Calculation for $1 \%$ HDPE

Weight $=0.0386 * 1 / 100 * 100 * 100 * 100 * 0.055=21.23$ grams.

Weight of fibres for varying \% of HDPE for $1 \mathrm{~m}^{3}$ of concrete 


\begin{tabular}{|c|c|c|}
\hline $\begin{array}{c}\text { Sl. } \\
\text { No. }\end{array}$ & \% of HDPE & Wt. of HDPE $\left(\mathbf{g m} / \mathbf{m}^{\mathbf{3}}\right)$ \\
\hline 1. & 0.5 & 275 \\
\hline 2. & 1.0 & 550 \\
\hline 3. & 1.5 & 825 \\
\hline 4. & 2.0 & 1100 \\
\hline 5. & 2.5 & 1375 \\
\hline 6. & 3.0 & 1650 \\
\hline 7. & 3.5 & 1925 \\
\hline 8. & 4.0 & 2200 \\
\hline 9. & 4.5 & 2475 \\
\hline 10. & 5.0 & 2750 \\
\hline 11 & 5.5 & 3025 \\
\hline 12. & 6.0 & 3300 \\
\hline
\end{tabular}

For $1 \% \mathrm{HDPE}$

$0.0386 \mathrm{M} 3$ of concrete weight of jute is 21.23 grams

$1 \mathrm{cu} . \mathrm{m}$ the weight of jute $=(1 / 0.0386) \times 21.23=550 \mathrm{grams} / \mathrm{cu} . \mathrm{M}$

\section{RESULTS \& DISCUSSION}

28 - Days Compressive Strength with varying \% of HDPE by volume

\begin{tabular}{|c|c|c|c|c|c|c|c|}
\hline \multirow{2}{*}{$\begin{array}{l}\text { Sl. } \\
\text { No. }\end{array}$} & \multirow{2}{*}{ ID No } & \multirow{2}{*}{$\begin{array}{l}\% \text { of } \\
\text { HDPE }\end{array}$} & \multicolumn{3}{|c|}{ Ultimate Load (kN) } & \multirow{2}{*}{$\begin{array}{l}\text { Average Load } \\
(\mathrm{kN})\end{array}$} & \multirow{2}{*}{$\begin{array}{l}\text { Average } \\
\text { strength } \\
\left(\mathrm{N} / \mathrm{mm}^{2}\right)\end{array}$} \\
\hline & & & Sample-1 & Sample-2 & Sample-3 & & \\
\hline 1 & S1 & 0.0 & 930 & 990 & 930 & 950.0 & 42.23 \\
\hline 2 & S2 & 0.5 & 930 & 970 & 1000 & 966.7 & 42.97 \\
\hline 3 & S3 & 1.0 & 1000 & 950 & 975 & 975.0 & 43.34 \\
\hline 4 & S4 & 1.5 & 950 & 1020 & 980 & 983.3 & 43.71 \\
\hline 5 & S5 & 2.0 & 970 & 1000 & 1000 & 990.0 & 44.00 \\
\hline 6 & S6 & 2.5 & 1000 & 940 & 960 & 996.7 & 44.3 \\
\hline 7 & S7 & 3.0 & 1000 & 1040 & 990 & 1010 & 44.90 \\
\hline 8 & S8 & 3.5 & 1000 & 1040 & 990 & 1010.0 & 44.90 \\
\hline 9 & S9 & 4.0 & 950 & 980 & 995 & 975.0 & 43.34 \\
\hline 10 & $\mathrm{~S} 10$ & 4.5 & 960 & 920 & 865 & 915.0 & 40.67 \\
\hline 11 & S11 & 5.0 & 800 & 850 & 825 & 825.0 & 36.67 \\
\hline 12 & S12 & 5.5 & 710 & 800 & 730 & 746.7 & 33.20 \\
\hline 13 & S13 & 6.0 & 700 & 680 & 720 & 700.0 & 31.10 \\
\hline
\end{tabular}


Combination of 28 Days Compressive Strength of HDPE Fiber Reinforced Concrete (0\% and 3\%)

\begin{tabular}{|c|c|c|c|c|}
\hline $\begin{array}{l}\text { Sl. } \\
\text { No. }\end{array}$ & $\%$ of HDPE & Load $(\mathrm{kN})$ & $\begin{array}{c}\text { Average Load } \\
(\mathrm{kN})\end{array}$ & Average Strength $\left(\mathrm{N} / \mathrm{mm}^{2}\right)$ \\
\hline 1 & 0.0 & 930 & \multirow{3}{*}{950.0} & \multirow{3}{*}{42.23} \\
\hline 2 & 0.0 & 990 & & \\
\hline 3 & 0.0 & 930 & & \\
\hline 4 & 3.0 & 1000 & \multirow{3}{*}{1010} & \multirow{3}{*}{44.90} \\
\hline 5 & 3.0 & 1040 & & \\
\hline 6 & 3.0 & 990 & & \\
\hline
\end{tabular}

Modulus of elasticity of M-30 grade of concrete with $0 \%$ of HDPE.

\begin{tabular}{|c|c|c|c|c|c|c|c|c|}
\hline $\begin{array}{l}\text { Sl. } \\
\text { No }\end{array}$ & $\begin{array}{l}\text { Load } \\
\text { (kn) }\end{array}$ & $\begin{array}{c}\delta \\
\mathrm{C} 1\end{array}$ & $\begin{array}{c}\Delta \\
\Delta \\
\mathrm{C} 2\end{array}$ & $\begin{array}{c}\delta \\
\mathrm{C} 3\end{array}$ & $\begin{array}{c}\delta \\
\text { avg }\end{array}$ & $\begin{array}{c}\text { Stress } \\
(\mathbf{f}=\mathbf{P} / \mathbf{A})\end{array}$ & Strain $(\mathrm{e}=\mathbf{\delta} / \mathrm{l})$ & $E=f / e$ \\
\hline 1 & 0 & 0 & 0 & 0 & 0 & 0 & 0 & 0 \\
\hline 2 & 10 & 0.6 & 0.6 & 0.6 & 0.6 & 0.57 & $0.6^{*} 10^{-5}$ & 95000 \\
\hline 3 & 20 & 1.2 & 1 & 1.2 & 1.13 & 1.13 & $1.13 * 10^{-5}$ & 100000 \\
\hline 4 & 30 & 1.8 & 1.4 & 1.8 & 1.67 & 1.7 & $1.67 * 10^{-5}$ & 101796 \\
\hline 5 & 40 & 2.4 & 2.2 & 2.4 & 2.33 & 2.26 & $2.33 * 10^{-5}$ & 96996 \\
\hline 6 & 50 & 3.2 & 2.8 & 3.3 & 3.1 & 2.83 & $3.1^{*} 10^{-5}$ & 91290 \\
\hline 7 & 60 & 4.0 & 3.6 & 4 & 3.87 & 3.4 & $3.87 * 10^{-5}$ & 87855 \\
\hline 8 & 70 & 4.8 & 4 & 4.8 & 4.53 & 3.96 & $4.53 * 10^{-5}$ & 87417 \\
\hline 9 & 80 & 5.6 & 4.8 & 5.6 & 5.33 & 4.53 & $5.33 * 10^{-5}$ & 84991 \\
\hline 10 & 90 & 6.8 & 5.6 & 6.9 & 6.43 & 5.1 & $6.43^{*} 10^{-5}$ & 79316 \\
\hline 11 & 100 & 8.0 & 6 & 8 & 7.33 & 5.66 & $7.33 * 10^{-5}$ & 77217 \\
\hline 12 & 110 & 8.8 & 6.6 & 9 & 8.13 & 6.23 & $8.13 * 10^{-5}$ & 76630 \\
\hline 13 & 120 & 9.6 & 7.2 & 10 & 8.93 & 6.8 & $8.93 * 10^{-5}$ & 76148 \\
\hline 14 & 130 & 10.2 & 7.8 & 11.1 & 9.7 & 7.36 & $9.7 * 10^{-5}$ & 75876 \\
\hline 15 & 140 & 11.2 & 8.4 & 12.2 & 10.6 & 7.92 & $10.6^{*} * 10^{-5}$ & 74717 \\
\hline 16 & 150 & 11.8 & 9 & 13.4 & 11.4 & 8.49 & $11.4^{*} 10^{-5}$ & 74474 \\
\hline 17 & 160 & 12.8 & 9.6 & 14.6 & 12.33 & 9.06 & $12.33^{*} 10^{-3}$ & 73479 \\
\hline 18 & 170 & 13.4 & 10.2 & 15.8 & 13.13 & 9.62 & $13.13^{*} 10^{-5}$ & 73267 \\
\hline 19 & 180 & 14.2 & 10.8 & 17 & 14 & 10.19 & $14.0^{*} 10^{-5}$ & 72786 \\
\hline 20 & 190 & 15.0 & 12 & 18.3 & 15.1 & 10.75 & $15.1 * 10^{-5}$ & 71192 \\
\hline 21 & 200 & 16.0 & 12.8 & 19.6 & 16.1 & 11.32 & $16.1 * 10^{-5}$ & 70311 \\
\hline 22 & 210 & 16.8 & 13.4 & 20.9 & 16.46 & 11.88 & $16.46^{*} 10^{-5}$ & 72175 \\
\hline 23 & 220 & 17.6 & 14.2 & 22.3 & 18.03 & 12.45 & $18.03 * 10^{-5}$ & 69052 \\
\hline 24 & 230 & 18.2 & 15 & 23.6 & 18.93 & 13.02 & $18.93^{*} 10^{-5}$ & 68780 \\
\hline 25 & 240 & 19.2 & 15.4 & 24.8 & 19.8 & 13.58 & $19.8 * 10^{-5}$ & 68586 \\
\hline 26 & 250 & 20 & 15.6 & 26.2 & 20.6 & 14.15 & $20.6^{*} 10^{-5}$ & 68689 \\
\hline 27 & 260 & 21 & 15.8 & 27.6 & 21.47 & 14.71 & $21.47^{*} 10^{-5}$ & 68514 \\
\hline 28 & 270 & 21.8 & 16.5 & 29 & 22.43 & 15.28 & $22.43^{*} 10^{-5}$ & 68123 \\
\hline 29 & 280 & 22.8 & 17 & 30.4 & 23.4 & 15.85 & $23.4^{*} \cdot 10^{-5}$ & 67735 \\
\hline 30 & 290 & 23.6 & 17.8 & 31.9 & 24.43 & 16.41 & $24.43^{*} 10^{-5}$ & 67172 \\
\hline 31 & 300 & 25 & 19 & 33.3 & 25.77 & 16.98 & $25.77^{*} 10^{-5}$ & 65891 \\
\hline 32 & 310 & 25.8 & 19.8 & 34.8 & 26.8 & 17.54 & $26.8 * 10^{-5}$ & 65448 \\
\hline 33 & 320 & 26 & 20.6 & 36.4 & 27.67 & 18.11 & $27.67 * 10^{-5}$ & 65450 \\
\hline 34 & 330 & 27 & 21.5 & 38 & 28.83 & 18.67 & $28.83 * 10^{-5}$ & 64759 \\
\hline
\end{tabular}


Table - 1: Modulus of elasticity of M-30 grade of concrete with 3.0\% of HDPE.

\begin{tabular}{|c|c|c|c|c|c|c|c|c|}
\hline Sl. No. & $\begin{array}{c}\text { Load } \\
\text { (kn) }\end{array}$ & $\begin{array}{c}\delta \\
\mathrm{C} 1 \\
\end{array}$ & $\begin{array}{c}\delta \\
\mathrm{C} 2 \\
\end{array}$ & $\begin{array}{c}\delta \\
\mathrm{C} 3 \\
\end{array}$ & $\begin{array}{c}\delta \\
\text { avg } \\
\end{array}$ & $\begin{array}{c}\text { Stress } \\
(\mathrm{f}=\mathrm{P} / \mathrm{A})\end{array}$ & Strain $(e=\delta / l)$ & $\mathrm{E}=\mathrm{f} / \mathrm{e}$ \\
\hline 1 & 0 & 0 & 0 & 0 & 0 & 0 & 0 & 0 \\
\hline 2 & 10 & 0.4 & 0.4 & 0.5 & 0.43 & 0.57 & $0.43 * 10^{-5}$ & 132558 \\
\hline 3 & 20 & 1 & 0.8 & 1 & 0.9 & 1.13 & $0.9 * 10^{-5}$ & 125555 \\
\hline 4 & 30 & 1.6 & 1.4 & 1.8 & 1.6 & 1.7 & $1.6 * 10^{-5}$ & 106250 \\
\hline 5 & 40 & 2.4 & 2 & 2.5 & 2.3 & 2.26 & $2.3 * 10^{-5}$ & 98261 \\
\hline 6 & 50 & 3.2 & 2.8 & 3.2 & 3.1 & 2.83 & $3.1 * 10^{-5}$ & 91290 \\
\hline 7 & 60 & 4 & 3.4 & 4 & 3.8 & 3.4 & $3.8 * 10^{-5}$ & 89474 \\
\hline 8 & 70 & 4.8 & 3.8 & 4.8 & 4.5 & 3.96 & $4.5 * 10^{-5}$ & 88000 \\
\hline 9 & 80 & 5.6 & 4.6 & 5.5 & 5.2 & 4.53 & $5.2 * 10^{-5}$ & 87115 \\
\hline 10 & 90 & 6.4 & 5.8 & 6.2 & 6.1 & 5.1 & $6.1 * 10^{-5}$ & 83606 \\
\hline 11 & 100 & 7 & 6.6 & 7 & 6.9 & 5.66 & $6.9 * 10^{-5}$ & 82029 \\
\hline 12 & 110 & 7.8 & 7.4 & 7.8 & 7.7 & 6.23 & $7.7^{*} 10^{-5}$ & 80909 \\
\hline 13 & 120 & 8.6 & 8 & 8.8 & 9.1 & 6.8 & $9.1 * 10^{-5}$ & 77474 \\
\hline 14 & 130 & 9.6 & 8.8 & 10 & 9.5 & 7.36 & $9.5 * 10^{-5}$ & 76154 \\
\hline 15 & 140 & 10.5 & 9.8 & 11 & 10.4 & 7.92 & $10.4 * 10^{-5}$ & 75133 \\
\hline 16 & 150 & 11.2 & 10.8 & 12 & 11.3 & 8.49 & $11.3^{*} 10^{-5}$ & 74725 \\
\hline 17 & 160 & 12.2 & 11.6 & 13 & 12.3 & 9.06 & $12.3 * 10^{-5}$ & 73658 \\
\hline 18 & 170 & 13 & 12.4 & 14 & 13.1 & 9.62 & $13.1 * 10^{-5}$ & 73435 \\
\hline 19 & 180 & 14 & 13.2 & 15 & 14.1 & 10.19 & $14.1 * 10^{-5}$ & 72269 \\
\hline 20 & 190 & 15 & 14 & 16 & 15 & 10.75 & $15.0 * 10^{-5}$ & 71667 \\
\hline 21 & 200 & 16 & 15.5 & 17.2 & 16.2 & 11.32 & $16.2 * 10^{-5}$ & 69877 \\
\hline 22 & 210 & 17 & 17 & 18 & 17.3 & 11.88 & $17.3 * 10^{-5}$ & 68671 \\
\hline 23 & 220 & 18 & 17.8 & 19 & 18.3 & 12.45 & $18.3 * 10^{-5}$ & 68033 \\
\hline 24 & 230 & 19.8 & 19 & 20 & 19.6 & 13.02 & $19.6 * 10^{-5}$ & 66429 \\
\hline 25 & 240 & 21 & 19.8 & 21 & 20.6 & 13.58 & $20.6 * 10^{-5}$ & 65922 \\
\hline 26 & 250 & 22.2 & 20.8 & 22.2 & 21.7 & 14.15 & $21.7 * 10^{-5}$ & 65207 \\
\hline 27 & 260 & 24 & 21.6 & 23 & 22.9 & 14.71 & $22.9 * 10^{-5}$ & 64236 \\
\hline 28 & 270 & 25.2 & 22.6 & 24 & 23.6 & 15.28 & $23.6 * 10^{-5}$ & 64746 \\
\hline 29 & 280 & 26.2 & 23.6 & 25 & 26.1 & 15.85 & $26.1 * 10^{-5}$ & 60728 \\
\hline 30 & 290 & 27.4 & 24.6 & 26.2 & 27.3 & 16.41 & $27.3 * 10^{-5}$ & 60110 \\
\hline 31 & 300 & 29.2 & 25.5 & 27.2 & 28.3 & 16.98 & $28.3 * 10^{-5}$ & 60000 \\
\hline 32 & 310 & 31 & 27 & 28.5 & 29.7 & 17.54 & $29.7 * 10^{-5}$ & 59057 \\
\hline 33 & 320 & 32.2 & 29.2 & 29.5 & 31.1 & 18.11 & $31.1 * 10^{-5}$ & 58232 \\
\hline 34 & 330 & 33.4 & 31 & 31 & 31.8 & 18.67 & $31.8 * 10^{-5}$ & 58711 \\
\hline 35 & 340 & 35 & 32.4 & 32.5 & 33.3 & 19.24 & $33.3 * 10^{-5}$ & 57777 \\
\hline 36 & 350 & 36.5 & 33.8 & 34 & 34.8 & 19.81 & $34.8 * 10^{-5}$ & 56925 \\
\hline 37 & 360 & 38.2 & 35.2 & 35.5 & 36.3 & 20.37 & $36.3 * 10^{-5}$ & 56116 \\
\hline
\end{tabular}




\section{CONCLUSION}

1. The HDPE concrete has shown slight improvement in all properties when compared with the conventional concrete.

Compressive strength by $10.63 \%$

Split tensile strength by $15.76 \%$

Flexural strength by $13.9 \%$

2. HDPE fibres are mainly drawn from cement bags, the entire construction site will be free from its own nbiodegradable waste, as one cum of HDPE fibre reinforced concrete requires 50 bags at $3.0 \%$ of fibres by volume.

3. HDPE fibre reinforced concrete it durable as it improved the strength gradually by $11.25 \%$ at 7 days and $10.63 \%$ increase after 28 days.

4. Jute plastic cement bags can be used immediately after using the cement in the site.

5. NBD - FRC can be used in sewerage pipes, manhole covers, compound walls, retaining walls etc.
1. Further work can be investigated for durability for individual and mixture of fibres.

2. Further study by replacing the same amount of cement by volume.

3. Further study on scc

\section{LIMITATIONS}

Aspect ratio should be 100 to 150 .

\section{REFERENCES}

[1]. A.K. Shrivastava, A.K. Sahu, M.Shukla and A.Ksaclan "Impact resistance of fiber reinforced concrete " Advances in cement in concrete $-\mathrm{pg}-80$.

[2]. Ashour S.A. and Wafa, F.F (1993), "Flexural behavior of high strength fiber reinforced concrete beams". ACI

Structural Journal, May-June 1993, Vol. 90, No.3, PP 279 287. Eight high - strength concrete beams with different

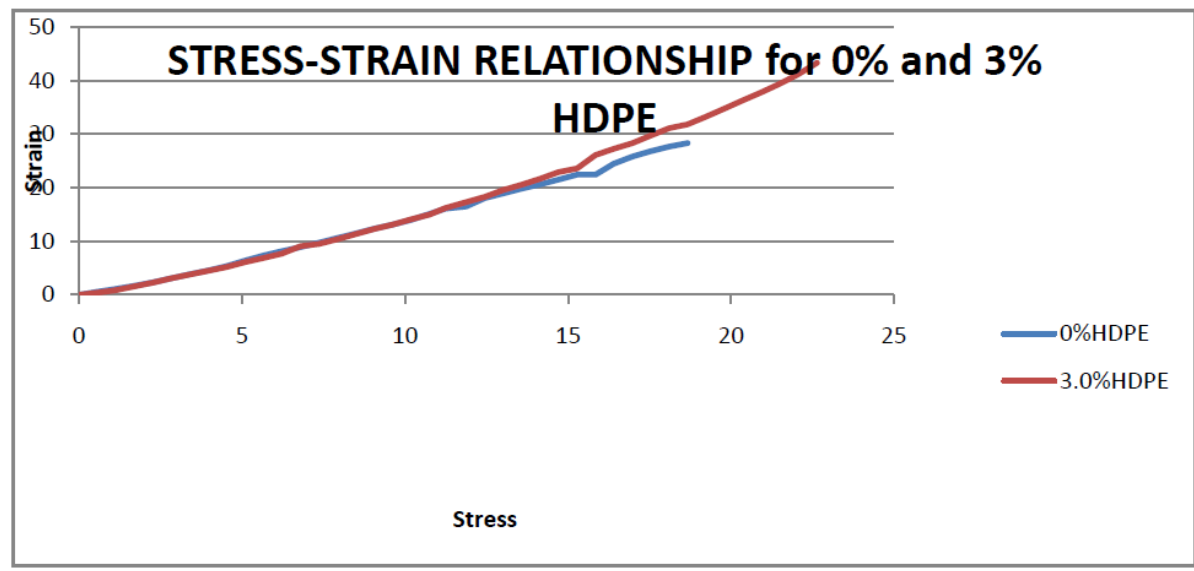

28 days Compressive Strength with varying \% of HDPE

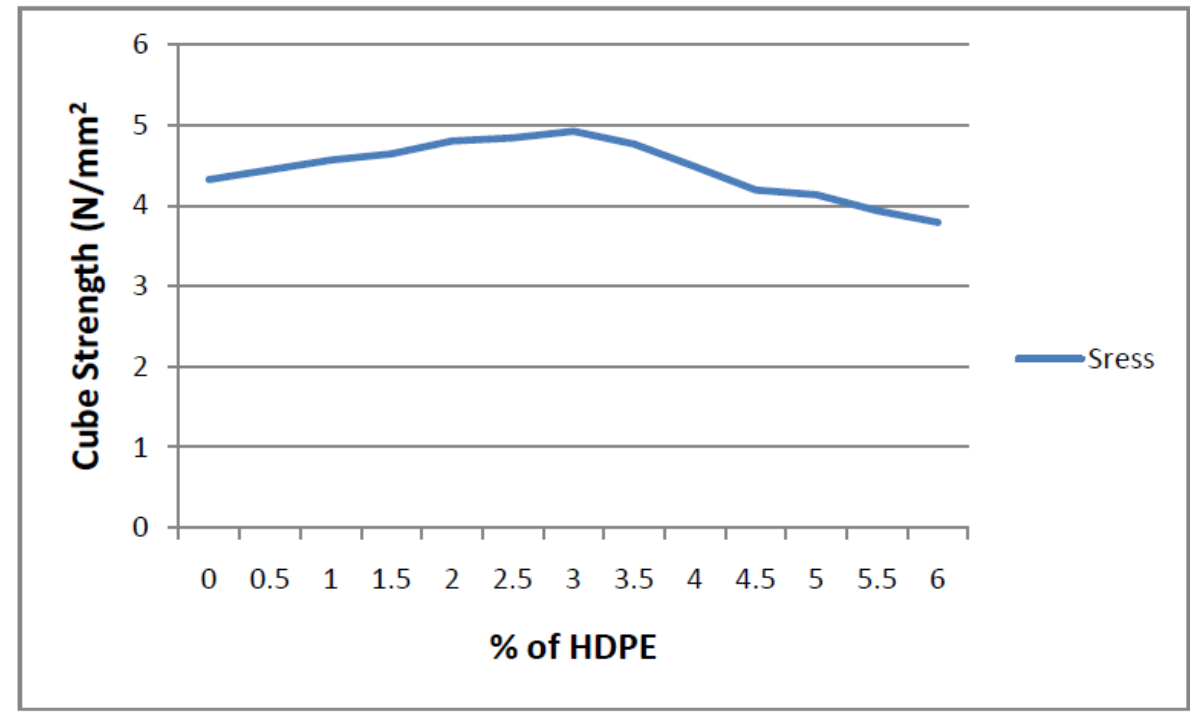

7 days Compressive Strength with varying \% of HDPE 